\title{
Describing objectively measured physical activity levels, patterns, and correlates in a cross sectional sample of infants and toddlers from South Africa
}

Alessandra Prioreschi ${ }^{1 *}\left(\mathbb{D}\right.$, Soren Brage ${ }^{2}$, Kylie D. Hesketh $^{3}$, Jill Hnatiuk ${ }^{3}$, Kate Westgate ${ }^{2}$ and Lisa K. Micklesfield ${ }^{1}$

\begin{abstract}
Background: Physical activity is considered to have health benefits across the lifespan but levels, patterns, and correlates have not been well described in infants and toddlers under the age of two years.

Methods: This study aimed to describe objectively and subjectively measured physical activity in a group of South African infants aged 3- to 24-months $(n=140)$, and to investigate individual and maternal correlates of physical activity in this sample. Infants' physical activity was measured using an Axivity AX3 wrist-worn accelerometer for one week and the mean vector magnitude was calculated. In addition, mothers reported the average amount of time their infant spent in various types of activities (including in front of the TV), their beliefs about infants' physical activity, access to equipment in the home environment, and ages of motor development milestone attainment. Analysis of variance (ANOVA) and pair-wise correlations were used to test age and sex differences and associations with potential correlates.
\end{abstract}

Results: There were significant age and sex effects on the distribution of time spent at different physical activity intensities (Wilks' lambda $=0.06, p<0.01$ ). In all cases, the trend was for boys to spend more time in higher intensity physical activity and less time in lower intensity activity than girls; and for time spent in higher intensity activities to be higher in older children. Time spent outside was higher in boys, and this reached significance at 18-months $(F=3.84, p=0.02)$. Less concern around floor play was associated with higher physical activity at 12-months in females only $(p=0.03, r=0.54)$, and no other maternal beliefs were correlated with physical activity. The majority (94\%) of children were exceeding TV time recommendations. When controlling for age and sex, overall TV time was positively associated with BMl $z$-score $(\beta=0.01, p=0.05)$.

Conclusion: This study is the first to show sex and age differences in the patterns of physical activity, and to report on objectively measured and maternal reported physical activity and sedentary behaviour in the first two years of life in South Africa infants. Infants and toddlers should be provided with as many opportunities to be active through play as possible, and TV time should be limited.

Keywords: Infant, Physical activity, Television viewing, Accelerometery, South Africa

\footnotetext{
* Correspondence: Alessandra.Prioreschi@wits.ac.za

${ }^{1}$ MRCMITS Developmental Pathways for Health Research Unit, Department

of Paediatrics, School of Clinical Medicine, Faculty of Health Sciences,

University of Witwatersrand, Johannesburg, South Africa

Full list of author information is available at the end of the article
} 


\section{Background}

Physical activity has beneficial effects on health at all stages of the lifecourse. Data from higher income countries has shown that physical activity in early childhood (0-4 years) has beneficial effects on current and future health, and that sedentary behaviour may have a detrimental influence on body composition, which subsequently influences health outcomes [1-3]. Physical activity in the early years has also been shown to be beneficial for cognitive and motor development, and emotional and physical well-being [2]. Further, in a systematic review increased screen time has been shown to be associated with weight gain in children under age two [4]; and a metaanalysis of current evidence has shown that rapid weight gain in the first two years of life is associated with increased adiposity in later childhood [5]. However, there is a paucity of data describing objectively measured physical activity and sedentary behaviour at a very young age i.e. in the first two years of life [6].

The first two years of life have been shown to be critical determinants of adult behaviours and health risks [7], yet in South Africa no studies have objectively measured physical activity and sedentary behaviours in this period [7]. According to survey data, South Africans present with high sedentary time and low physical activity levels from a young age [8]. A high prevalence of overweight is already apparent in young children (ranging from $11 \%-14 \%$ in children aged 6 years [9], with $20 \%$ of toddlers at age two presenting with overweight [8]). Similar to international findings, approximately half of South African children are meeting age specific physical activity recommendations [9]. Internationally, studies have shown that participation in sedentary behaviors (mainly television (TV) viewing) is already higher than recommended in the first two years of life [4, 10, 11]; and similarly excessive sedentary time (73\% of the day) is apparent in South African children as young as 4 years old [12].

Correlates of activity levels in the first two years of life have not been extensively described [6]. A systematic review of the cross sectional correlates of physical activity in preschool children (aged 2-5 years) conducted in 2008 showed that being male, spending more time outdoors, and having an active parent, were correlated with being more active [13]. A more recent systematic review of longitudinal observational studies and controlled trials conducted in 2016 showed that predictors of increased physical activity in 0-6 year olds included parental monitoring of their children's activity, maternal role modeling; and there was some inconsistent evidence that boys had higher physical activity than girls [14]. This systematic review reported that very few studies had been conducted in healthy children under age two, or in developing countries (with none conducted in Africa), however recently studies have been conducted measuring physical activity response to dietary treatment or supplementation in undernourished toddlers in Africa $[15,16])$. Studies on infants and toddlers have consistently shown age to be associated with increased sedentary behaviour and with more time spent in higher intensity physical activity [17-20], yet it is likely that developmental stage (rather than chronological age) will impact on ability to participate in certain types and intensities of activity, thus presenting a bidirectional relationship between developmental stage and physical activity [21]. Furthermore, maternal factors such as mother's TV time and physical activity were associated with toddler sedentary time and physical activity, respectively $[17,18,20]$. Mother's education level (higher), race (minority), and the home environment; and time toddlers spent being active with their mother and with other toddlers, have also been correlated with toddler sedentary time and physical activity respectively [17-19]. In toddlers who were walking (aged 12- to 36-months), boys were more active than girls [20].

The need to measure and examine levels of physical activity and sedentary time in the first two years of life is clear. Furthermore, correlates of these behaviours should be examined in order to intervene effectively. Preliminary data and data from older children suggest that the home environment and mother's behaviours, and child sex and age (and/or developmental stage) are important correlates of physical activity and sedentary behaviour. Therefore this study aims to 1) describe levels and patterns of physical activity and sedentary behaviour in a group of South African infants/toddlers aged 3- to 24months using objective and subjective data, and 2) investigate and describe individual and maternal correlates of objectively measured physical activity.

\section{Methods}

\section{Participants and procedures}

Participants (convenience sample of 152 mother and child pairs) were recruited from the Chris Hani Baragwanath Academic Hospital, MRC/Wits Developmental Pathways for Health Research Unit in Soweto, South Africa. Soweto is over $200 \mathrm{~km}^{2}$ in size with over 1.2 million inhabitants. Fathers in Soweto often have little to no involvement in childcare [22], and therefore only mothers were considered in this study. In most cases, mothers had been recruited from the Chris Hani Baragwanath Academic Hospital (which is the largest public hospital in South Africa and is thus likely to provide a sample of mothers representative of the Soweto community) for participation in a pregnancy and infancy study (M130905) at the research unit, and were invited telephonically to participate in the current study if their child was within 2 weeks of 3-, 6-, 12-, 18-, or 24-months of age (information obtained via pregnancy study birth records). For the purposes of this paper, children of all ages from this sample will be referred 
to as "infants". Participants were excluded if infants had any diagnosed developmental abnormalities that may affect normal movement or development. Mothers were required to read and sign informed consent and assent documents for themselves and on behalf of their infant, and were free to withdraw from the study at any time. Ethical approval for this study was provided by the University of the Witwatersrand Human Research Ethics Committee (M150632).

Participants were required to visit the research unit twice. At the first visit all demographic information was collected, questionnaires completed, measurements taken, and infants were fitted with an accelerometer and mothers were provided with a sleep diary to complete for their infant. At the second visit, one week later, participants were required to return the accelerometers and sleep diaries. If participants were unable to return the devices, researchers collected the devices from participants' homes.

\section{Outcome measures Physical activity}

Infants' physical activity was objectively measured using an Axivity AX3 wrist worn accelerometer (Axivity Ltd., Newcastle-upon-Tyne, UK). These monitors allow for transparent data processing analysis due to the opensource software platform and unforced sampling of raw measurement data [23, 24]. Furthermore, they are small enough to be worn on the wrist of infants from 3 months. Monitors were initialised (using the open source OmGui software, Open Movement, UK) to capture triaxial acceleration data at $100 \mathrm{~Hz}$ with a dynamic range of $+/-8 \mathrm{~g}$. The monitors were worn in a specially designed fabric band (Open Lab, Newcastle, UK), which is waterproof, breathable, flexible and safe. This band was designed and piloted by the authors and Open Lab, and has been successfully used and tested for feasibility in a South African sample of infants (manuscript under review [25]). Mothers were instructed not to remove the band from their infant's wrist for a seven-day period. Following download (OmGui, Open Movement, UK), the raw acceleration data were autocalibrated to gravity, using methods described elsewhere [26]; and vector magnitude (expressed in $\mathrm{mg}$ ) was calculated representing the magnitude of acceleration over the summarised time period [23]. A high-pass $(0.2 \mathrm{~Hz})$ and low-pass $(20 \mathrm{~Hz})$ frequency filter was then applied to the data in order to remove gravity, as well as high frequency noise [27]. Thus, the remaining data provided an approximation of acceleration due to human movement alone. Thereafter, non-wear was identified based on the deviation of each axis below $13 \mathrm{mg}$ for $>1 \mathrm{~h}$, and removed [23]. Remaining data was summarised to generate average acceleration $(\mathrm{mg})$, as well as time (hourly and weekly) spent in intensity thresholds (10 mg increments from 0 to $400 \mathrm{mg}$ ). During summation, non-wear periods were taken into account in order to minimize potential diurnal bias [28]. At least $45 \mathrm{~h}$ of wear time (equating to three days of at least $15 \mathrm{~h}$ data per day [29]) spread equally across the 24-h day (at least $4 \mathrm{~h}$ each day during morning, noon, afternoon, and night) were required for inclusion in the analysis.

\section{Correlates and covariates \\ Anthropometry and demographics}

Mothers were asked to report their date of birth and their infants' date of birth and sex. Infant age was calculated and categorised to the nearest month. Mothers' height was taken to the nearest $1 \mathrm{~mm}$ using a wallmounted stadiometer (Holtain, UK). Infant length was measured to the nearest $1 \mathrm{~mm}$ using an infantometer (Chasmors Ltd., UK). Weight of mothers and infants was measured to the nearest $0.1 \mathrm{~kg}$ using a digital scale (Dismed, USA). All anthropometry measurements were taken twice, by trained research staff according to standardised procedures, and the average of the two values was reported. Mother and infant body mass index (BMI) was calculated as (weight $(\mathrm{kg}) /$ height $\left.(\mathrm{m})^{2}\right)$. Infant weights, lengths and BMIs were converted to age-specific z scores according to the 2006/2007 World Health Organisation (WHO) growth standards [30] using the WHO Anthro software [31]. Mothers were asked to report how many hours per day they looked after their infant, and were considered primary caregivers if they reported looking after their infant for 8 or more hours per day.

\section{Questionnaire data}

All questionnaires used in this study were based on previously developed and reliability tested items [19], which were then adapted for language and setting based on pilot work conducted on mothers from Soweto. These adaptations mainly consisted of rewording sections that were not well understood in a South African context (i.e.: "...a baby that is very active" was changed to "...a baby that moves a lot").

\section{Infant activities}

Infant activities were assessed at 3-, 6-, 12-, and 18months. Mothers reported the amount of time (minutes) their infant spent in various activities, on an average day. Activities were age-specific, but included tummy time (only 3- and 6-months), time spent playing games with an adult, time spent being physically active with the mother, time spent on the floor, time spent with other babies of a similar age, time spent with older toddlers or children, time spent outside, time spent in front of a television (TV), time spent strapped to the mother's back (only 3-, 6- and 12-months), and time spent restrained (in a highchair, car chair, pushchair etc). Activities were pooled to determine total time restrained (i.e. sum of time 
spent strapped to mother's back, time spent in a highchair, car chair, pushchair, or cot), and total time free to play (i.e. sum of tummy time, time spent playing games with an adult, time spent being physically active with mother, time spent on the floor, time spent with other babies of a similar age, time spent with older toddlers or children, time spent outside). All activities are presented as minutes per day. Maternal reported time that infants spent in front of a TV was extracted from this questionnaire and used as proxy for TV time in order to examine potential correlates thereof. During the measurement period, mothers were asked to complete sleep diaries recording the time at which their infant was put to bed at night, and the time at which they were picked up from bed in the morning in order to provide an estimate of time in bed. If sleep diary data were missing, values were imputed based on trends for other infants' days and times.

\section{Maternal belief questionnaire}

Maternal beliefs about their infant's physical activity, and attitudes and intentions around their infant's physical activity and TV viewing, were assessed at 6- and 12months using a 24-item questionnaire with a 4-point likert-type scale and response options: $1=$ strongly agree to $4=$ strongly disagree. Based on previous studies [19], 7 factor variables were created, including physical activity knowledge (7 questions examining the importance of physical activity for infant health and development), positive views about physically active children (3 questions examining maternal perceptions of active children), negative views about physically active children (4 questions examining maternal fears and concerns about physically active children), physical activity optimism (3 questions examining the anticipated ease of engaging children in physical activity), self-efficacy for promoting physical activity (3 questions examining mothers' confidence for promoting physical activity), future expectations around infant's physical activity and TV viewing (2 questions examining maternal expectations of infant's future physical activity and TV viewing behaviours), and floor play concerns (2 questions examining perceptions of safety of floor play). Scores for each factor variable were then generated by averaging the item scores within each factor, where a higher score indicates lower agreement with the factor variable. The internal consistency in the present sample (mean Cronbach alpha $=0.60$ ) for these factor variables was acceptable and comparable to previous studies [19], with only one factor variable (positive views about physical activity) scoring below 0.60 .

\section{Home environment questionnaire}

Mothers of infants aged 3-, 6-, 12-, and 18-months were asked to report, using a 4 point likert-type scale, whether their child had access to, or was likely to have access to age-specific toys and equipment (such as balls, push toys, bicycles, etc) in the home or at nearby facilities within the next year. Each response was coded as "3" if mothers responded that they had the equipment already, " 2 " if they were very likely to get it within the next year, "1" if mothers responded they would possibly get the equipment in the next year, and " 0 " if mothers responded that they were unlikely to get the equipment within the next year. Thereafter a sum of the responses for each item was created, with a higher score indicating better access to equipment or intention to provide access, and a lower score indicating no access and/or less intention to provide access.

\section{Developmental milestones}

Mothers of infants aged 3-, 6-, 12-, and 18-months were asked to report whether their infant had attained specific motor development milestones according to the age of the child (based on the CDC recommendations: www.cdc.gov/ ActEarly). Milestones included: holding up head at 3months; sitting, crawling, and rolling to both sides at 6months; crawling, pulling up to stand, and starting to walk at 12-months; and walking and running at 18-months. Milestones were recorded as attained/not attained. Thereafter, infants were categorised as either not yet mobile (this assumed all 3-month infants, as well as those aged 12months who were not yet crawling); crawling (if 18-month infants were not yet walking/running they were presumed to be crawling); or walking/running (all infants aged 24-months were presumed to be walking/running). Assumptions were based on WHO reported ages for milestone attainment [32].

\section{Statistical analysis}

All statistical analyses were conducted using Stata 13 for Mac. Participant characteristics and all questionnaire data were summarised by age and presented as mean (SD), n (\%), or median (IQR). Since there is no consensus on intensity thresholds for sedentary, light, moderate, and vigorous activities in non-ambulatory infants (which made up the majority of the sample), objectively measured infant physical activity intensity distribution was presented as time spent in each systemic acceleration category from 0 to $400 \mathrm{mg}$ by age and sex. Furthermore, hourly mean vector magnitude (diurnal patterns) of activity were compared between ages, sex, and developmental stages. These differences were tested using multivariate analysis of variance (ANOVA) through Wilks' lambda test. Two-way ANOVAs, linear regressions, and students' unpaired $\mathrm{t}$-tests were used to test age and sex differences in the time spent in various activities as reported by mother, as well as for objectively measured physical activity. Thereafter, pairwise correlations were run by age for maternal variables (home environment, maternal BMI and 
age, maternal-reported infant activities, and maternal beliefs factor variables) on infant objectively measured physical activity. Regressions were also run for potential correlates of maternal reported infant TV time controlling for age and sex, and margins were calculated. Chi-squared tests were used to determine the proportion of infants meeting TV time guidelines according to developmental stage. A $p<0.05$ was considered significant in all cases.

\section{Results}

Of the 152 mother-infant pairs recruited for the study, six children did not have sufficient monitor wear time and were excluded from analyses. A further six infants lost their accelerometers or had missing data files. Participant characteristics for the remaining 140 participants with complete data are shown in Table 1. Although weight and length displayed were significantly higher between each consecutive age group except between 18- and 24-months $(p<0.01)$, there were no differences in BMI z-score by age $(p=0.61)$ or sex $(p=0.97)$, nor were there differences in weight $(p=0.14)$ or length $(p=0.47)$ between the boys and girls. Average reported time in bed was $10 \mathrm{~h} 18 \mathrm{~min}$ (range $7 \mathrm{~h} 29 \mathrm{~min}$ to $13 \mathrm{~h} 26 \mathrm{~min}$ ), and did not differ significantly by age or sex $(p=0.09$ and $p=0.91$ respectively). Most mothers (96\%) looked after their infants for $\geq 8 \mathrm{~h}$ per day. Boys were significantly more active than girls when comparing mean vector magnitude for the entire sample $(n=140)$ over the total wear period $(41(11) \mathrm{mg}$ vs $36(11) \mathrm{mg}, p<0.01)$, and this remained significant when categorising by age at 6 -, and 18-months. Total average physical activity for boys ranged from $21 \mathrm{mg}-83 \mathrm{mg}$; and for girls ranged from $17 \mathrm{mg}-61 \mathrm{mg}$. Physical activity had a direct linear relationship with age $(p<0.01)$ as shown in Fig. 1. Neither weight $(p=0.86)$, length $(p=0.55)$, nor BMI z-score $(p=1.00)$ were associated with physical activity when controlling for age and sex.

Figure 2 shows the time spent in each physical activity intensity distribution $(<160 \mathrm{mg}$ in Panel A and $>160 \mathrm{mg}$

Table 1 Characteristics of the study sample by age

\begin{tabular}{|c|c|c|c|c|c|}
\hline & $3 \mathrm{mo}(n=32)$ & $6 \mathrm{mo}(n=31)$ & $12 \mathrm{mo}(\mathrm{n}=31)$ & $18 \mathrm{mo}(n=18)$ & $24 \mathrm{mo}(n=28)$ \\
\hline Infant sex (\% boys) ${ }^{a}$ & $14(44)$ & $21(68)$ & $15(48)$ & $10(56)$ & $14(50)$ \\
\hline Infant weight $(\mathrm{kg})^{\mathrm{b}}$ & $5.8(0.8)$ & $7.7(1.2)$ & $9.0(1.0)$ & $9.9(1.5)$ & $10.8(1.2)$ \\
\hline Infant length $(\mathrm{cm})^{\mathrm{b}}$ & $58.7(5.1)$ & $65.0(2.7)$ & $72.9(3.6)$ & $79.3(4.3)$ & $82.3(3.9)$ \\
\hline Infant BMI z score ${ }^{b}$ & $0.2(2.5)$ & $0.6(1.6)$ & $0.3(1.3)$ & $-0.3(1.6)$ & $0.2(1.0)$ \\
\hline \multicolumn{6}{|c|}{ Attainment of milestones at given age $\mathrm{a}^{\mathrm{a}}$} \\
\hline Holding head & $21(66)$ & NA & NA & NA & NA \\
\hline Rolling & NA & $13(42)$ & NA & NA & NA \\
\hline Sitting & NA & $16(52)$ & NA & NA & NA \\
\hline Crawling & NA & $7(23)$ & $16(52)$ & NA & NA \\
\hline Pulling up & NA & NA & $14(45)$ & NA & NA \\
\hline Walking & NA & NA & $9(29)$ & $6(33)$ & $28(100)$ \\
\hline Running & NA & NA & NA & $4(22)$ & NA \\
\hline Maternal BMI $\left(\mathrm{kg} / \mathrm{m}^{2}\right)^{\mathrm{b}}$ & $28.5(9.1)$ & $27.8(6.7)$ & $29.5(7.0)$ & $27.5(8.0)$ & $27.3(6.7)$ \\
\hline Maternal age (years) ${ }^{b}$ & $27(5)$ & $31(6)$ & $29(5)$ & $32(6)$ & $29(7)$ \\
\hline Infant PA $(\mathrm{mg})^{c}$ & $23(17-28)$ & $30(24-34)$ & $36(30-39)$ & $39(35-45)$ & $44(37-51)$ \\
\hline Home Environment (score/48) ${ }^{\mathrm{b}}$ & $24(8)$ & $20(4)$ & $21(5)$ & $24(5)$ & NA \\
\hline \multicolumn{6}{|l|}{ Maternal Beliefs (score/4) ${ }^{\mathrm{a}}$} \\
\hline PA Knowledge & NA & $1.4(0.3)$ & $1.4(0.3)$ & NA & NA \\
\hline PA views & NA & $1.7(0.5)$ & $1.9(0.6)$ & NA & NA \\
\hline Negative PA views & NA & $3.0(0.5)$ & $2.8(0.6)$ & NA & NA \\
\hline PA optimism & NA & $1.4(0.4)$ & $1.5(0.5)$ & NA & NA \\
\hline Future expectations & NA & $2.5(0.8)$ & $2.6(0.7)$ & NA & NA \\
\hline Floor play concerns & NA & $3.0(0.8)$ & $3.0(0.6)$ & NA & NA \\
\hline Self efficacy & NA & $1.4(0.3)$ & $1.5(0.4)$ & NA & NA \\
\hline
\end{tabular}

$B M I$ - body mass index, PA - physical activity. At 24-months, all toddlers were assumed to be walking although not specifically reported at this time-point $N A$ Indicates that data was not acquired at the specific age ${ }^{a} \mathrm{n}(\%)$

${ }^{\mathrm{b}}$ All data are presented as mean(SD)

cor median(IQR) 


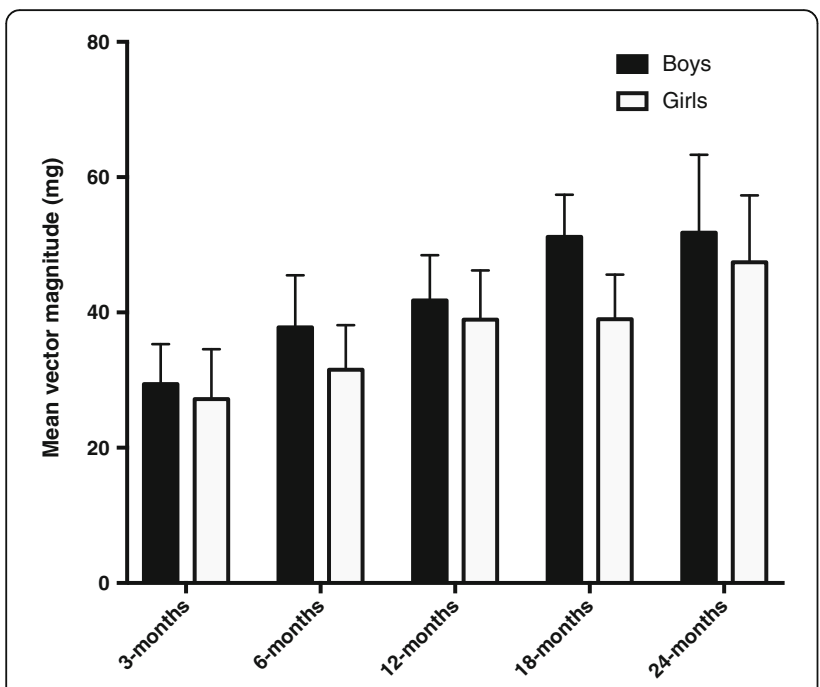

Fig. 1 Mean volume of physical activity by age and gender

in Panel B) by sex and age. There was a significant age and sex effect on intensity distributions (Wilks' lambda $=0.06, p<0.01)$. Girls were spending more time than boys in some lower intensities of activity $(<30 \mathrm{mg})$, and boys were spending more time than girls in most intensities $>70 \mathrm{mg}$, even when controlling for age $(p<0.05)$. There were also significant differences in time spent in each intensity of physical activity by age when controlling for sex $(p<0.05)$; as age increased, time spent in intensity of activity $<40 \mathrm{mg}$ was lower, and time spent in intensity $>70 \mathrm{mg}$ was higher. In all cases, the trend was for boys to spend more time in higher intensity physical activity and less time in lower intensity activity; and for time spent in higher intensity activities to be higher in the older age groups. When controlling for BMI z-score, weight or length, these relationships did not change (data not shown).

There was no significant interaction between developmental stage and sex (data not shown), however when dividing the data into developmental stage rather than age, boys spent more time in higher intensity activities than girls at each developmental stage, and both walkers and crawlers spent more time in activities $>50 \mathrm{mg}$ than infants who were not yet mobile.

Figure 3 shows the diurnal patterns of activity over an average 24-h period between boys and girls (Panel A), age groups (Panel B), and developmental stages (Panel C), respectively. Although patterns of activity were similar, boys had higher mean activity than girls, and this was significant at each hour between 6 am-11 am, and again from 3 pm-6 pm $(p<0.05)$, when controlling for age. Furthermore, when controlling for sex, as age increased, mean activity was higher and this was significant between 7 am-8 pm $(p<0.01)$. Between 2 am and 4 am, 3month infants were significantly more active than 24- month infants $(p<0.05)$. By visual interpretation of the data, as well as through examining these statistical effects, it seems as if most infants were becoming active at 6 am and decreasing activity from about $9 \mathrm{pm}$. Younger infants seem to experience a short, 2-h active period between 2 and $4 \mathrm{am}$. Peaks in activity are seen around $9 \mathrm{am}$, and again at around 3 pm- 6 pm with the highest activity accumulated between $5 \mathrm{pm}-6 \mathrm{pm}$. There was a significant interaction between age and sex on physical activity intensity distribution throughout the day (Wilks' Lamba $=0.20$, $p<0.01)$. These relationships remained largely unchanged when controlling for BMI $\mathrm{z}$-score (data not shown). Lastly, crawlers and walkers were more active during the day than infants who were not yet mobile, and in most cases walkers were more active than crawlers (except at $7 \mathrm{am}, 12 \mathrm{pm}$, and $7 \mathrm{pm}$ when crawlers were more active than walkers). There was a significant interaction between sex and developmental stage on distribution of physical activity intensity throughout the day (Wilks' Lamba $=0.34, p<0.01$ ); and these relationships remained significant when controlling for age (Wilks' Lambda $=0.13$, $p<0.01$ ), yet the effect of development was only significant at 7 am when crawlers were more active than walkers and not yet mobile infants.

Maternal reported time that infants spent in various activities (minutes per day) are shown in Table 2. Time spent outside was significantly higher in the older age groups and in boys (interaction effect: $\mathrm{F}=3.84, p=0.02$ ). Simple main effects showed that boys spent more time outside than girls at all ages except 12-months, and that this effect was significant at 18-months (mean(SD) time outside per day was 135(88) minutes in boys and 45(19) minutes in girls $(p=0.01)$. Age effects were also seen for time spent restrained in a high chair or in a cot while awake, which was lower as age increased $(p<0.05)$; and time spent playing, either in a play area or alone, which was higher as age increased $(p<0.01)$. The majority (94\%) of infants were exceeding TV time recommendations (no TV time recommended according to international guidelines $[33,34]$ ). This value improved with age from $3 \%$ meeting guidelines at 3-months to $17 \%$ meeting guidelines at 18-months, and was not different by sex.

When controlling for age and sex in the combined sample, overall TV time trended towards a positive association with BMI $z$-score $(\beta=0.01, p=0.05)$. Estimated regression margins suggest a dose response relationship between TV time and predicted BMI z-score, with only 0 min TV time predicting a negative BMI z-score. Meeting TV time guidelines was also associated with developmental stage $(p=$ $0.02)$, where a larger proportion of crawlers $(18 \%)$ met the guidelines than walkers $(2 \%)$ or not yet mobile infants (4\%). There was a significant interaction effect for age and development on meeting TV time guidelines $(p=0.04)$, yet age and development were not independently associated. 

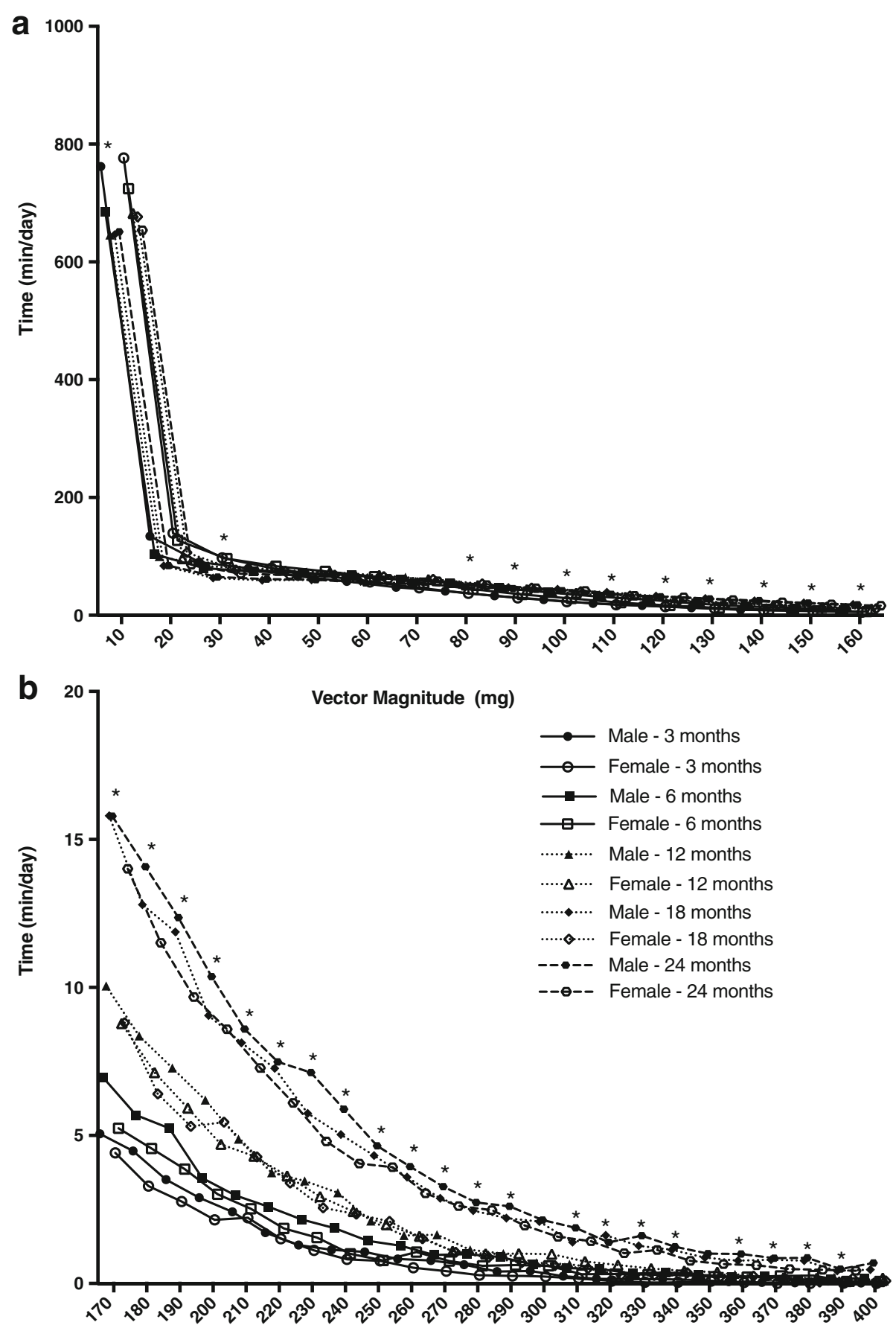

Fig. 2 Distribution of time spent $<160 \mathrm{mg}(\mathbf{a})$ and $>160 \mathrm{mg}$ (b) between sex and ages. * Indicates significant difference between boys and girls, controlling for age $(p<0.05)$

Pairwise correlations were run separately by age for maternal reported variables and objectively measured mean infant physical activity. At 6-months, playing games with an adult $(r=-0.40, p=0.03)$, and time in a cot $(r=-0.47, p<0.01)$ were both inversely associated with objectively assessed infant physical activity. When stratifying by sex, at 12-months, lower maternal concerns about floor play were positively associated with physical activity for girls only $(r=0.54, p=0.03)$. There were no significant correlates with objective physical activity at 3-, 18-, and 24-months; and mother's age, maternal beliefs about physical activity (except floor play concerns at 12-months in girls); and maternal reported infant tummy time, time restrained, time free, or time 

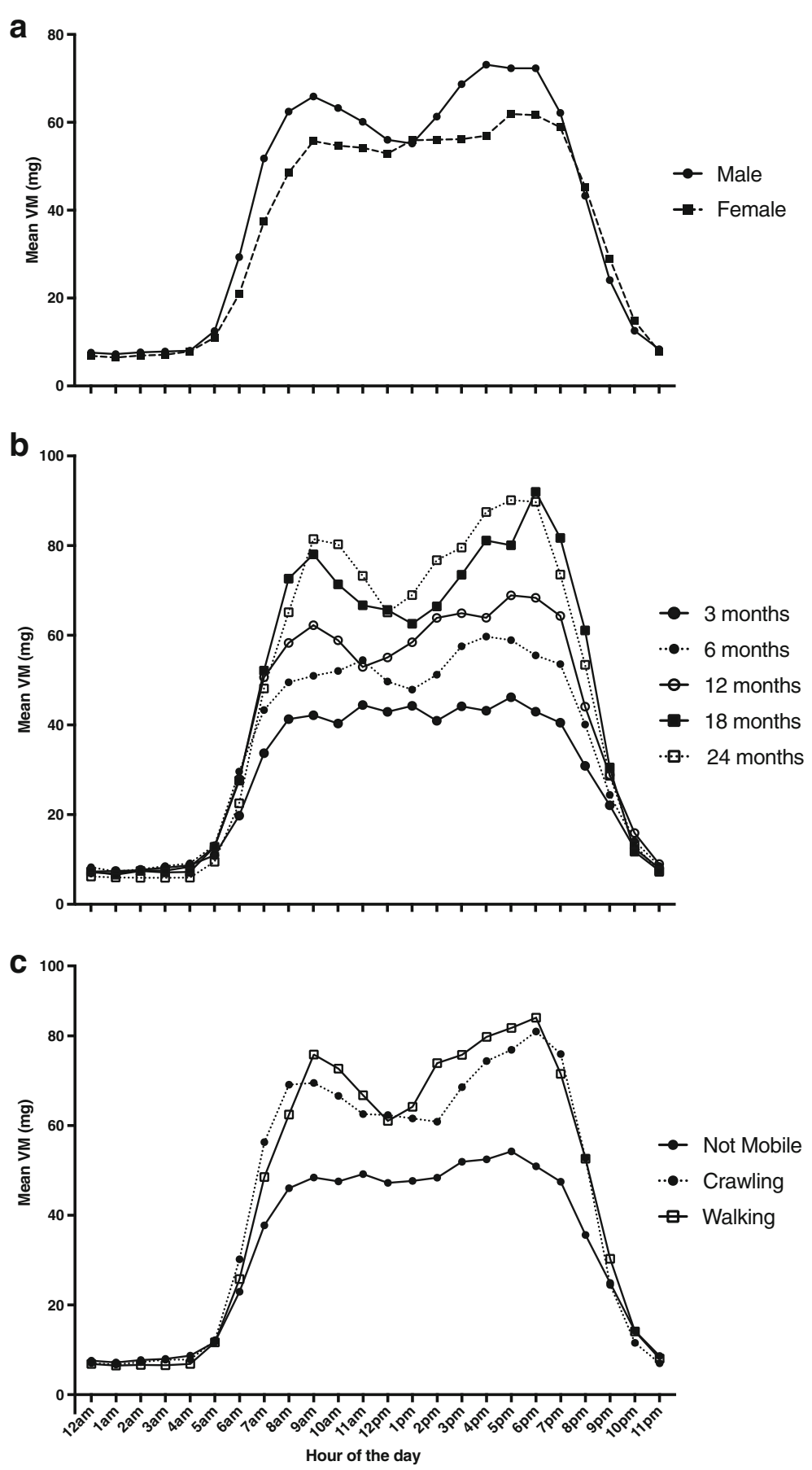

Fig. 3 Mean diurnal distributions of activity by sex (a), age (b), and developmental stage (c)

in bed were not correlated with objective physical activity at any age.

\section{Discussion}

This study is the first to objectively measure and describe physical activity levels and diurnal patterns in infants under age two in South Africa. Furthermore, few studies globally have thoroughly explored diurnal patterns and correlates of infant activities and behaviours in the first two years of life. We found that boys were significantly more active than girls throughout the day, regardless of age or developmental stage. These differences were evident as early as 3-months, and physical activity intensity increased significantly with age (regardless of developmental stage). Very few maternal or home environment variables were significantly associated with 
Table 2 Maternal reported infant activities by age

\begin{tabular}{|c|c|c|c|c|}
\hline Infant activity (min/day) & $3 \mathrm{mo}(n=32)$ & $6 \mathrm{mo}(n=31)$ & $12 \mathrm{mo}(n=31)$ & $18 \mathrm{mo}(n=18)$ \\
\hline \multicolumn{5}{|l|}{ Active time } \\
\hline Total time free to play & $60(25-93)$ & $90(45-130)$ & $150(120-240)$ & $158(115-210)$ \\
\hline Games with an adult & $20(5-30)$ & $15(2-45)$ & $10(5-30)$ & $18(4-30)$ \\
\hline Physical activity with mother & $8(4-30)$ & $10(5-30)$ & $15(5-60)$ & $18(10-45)$ \\
\hline Tummy time & $20(1-30)$ & $10(3-20)$ & NA & NA \\
\hline With older babies & $25(7-75)$ & $30(10-120)$ & $30(15-120)$ & $60(40-120)$ \\
\hline Outside & $0(0-0)$ & $0(0-30)$ & $30(0-60)$ & $60(50-120)$ \\
\hline With babies of same age & $0(0-0)$ & $0(0-2)$ & $0(0-15)$ & $28(0-60)$ \\
\hline Play area & $0(0-30)$ & $30(10-60)$ & $60(30-120)$ & $60(30-90)$ \\
\hline Floor play & $0(0-25)$ & $30(5-60)$ & $60(30-90)$ & $60(30-120)$ \\
\hline Playing alone & $30(15-60)$ & $30(10-45)$ & $60(30-60)$ & $30(20-30)$ \\
\hline \multicolumn{5}{|l|}{ Restrained/sedentary time } \\
\hline Total time restrained & $133(58-233)$ & $150(80-270)$ & $100(50-175)$ & $75(30-140)$ \\
\hline Pushchair & $0(0-0)$ & $0(0-10)$ & $0(0-30)$ & $0(0-0)$ \\
\hline Baby chair & $0(0-0)$ & $10(0-60)$ & NA & NA \\
\hline High chair & $0(0-20)$ & $20(0-60)$ & $10(2-30)$ & $10(4-20)$ \\
\hline Car chair & $20(3-60)$ & $30(5-30)$ & $30(10-60)$ & $35(15-60)$ \\
\hline Cot & $60(30-120)$ & $40(20-90)$ & $30(15-60)$ & $10(5-30)$ \\
\hline Strapped to back & $30(8-60)$ & $30(20-60)$ & $30(15-30)$ & NA \\
\hline TV time & 30 (20-90) & 30 (10-90) & $30(5-45)$ & $25(10-60)$ \\
\hline
\end{tabular}

Values are median(IQR). Data not available for 24-months

$N A$ Indicates that the activity was not asked about at the specific time-point

infant physical activity, yet it did seem that mothers provided boys with more opportunities to be active (eg. 18month boys spending 135 min playing outdoors compared to 18 -month girls spending only $45 \mathrm{~min}$ ). Importantly, almost all of the infants were exceeding TV time recommendations.

To our knowledge, this is the first study to show sex differences in physical activity intensity and diurnal patterns from such an early age, although these differences are widely reported in older children and adults [24, 35]. Various studies conducted internationally have found no differences in objectively measured physical activity between boys and girls at approximately 24-months [36-40], however it is important to note that none of these studies explored physical activity intensity distributions; and only three explored diurnal patterns of physical activity - one in 19-month toddlers in Australia [39], one in 6- to 23-month infants/toddlers from Burkino Faso with moderate acute malnutrition [40], and one in Ethiopian toddlers with severe acute malnutrition in which sex differences were not reported [15]. The Australian study found that boys were more active than girls in the morning, but observed no overall sex differences [39]. The Yameogo et al., study found no differences between boys and girls [40], yet it is difficult to compare these results, since morbidity was found to be associated with activity levels in the children with malnutrition, and findings therefore cannot be extrapolated to a healthy population of infants. Most of these above mentioned studies that showed no sex differences had reported activity levels according to intensity cut-offs that have only been validated in older children and may not be appropriate in this age group. A recent systematic review of predictors of change in physical activity in 0-6 year olds found evidence that boys may be more active than girls, yet this finding was not consistent and was not shown as early in life as in the current study [14]. The sex differences observed in the current study are intriguing, as they beg the question whether boys are inherently more active than girls, starting almost from birth, or whether these differences are dependent on differential opportunities provided to boy infants compared to girls. These sex differences were found regardless of age or developmental stage, and remained significant after controlling for body size. Furthermore, physical activity was not associated with any maternal reported variables, with the exception of maternal reports of time spent outdoors. Mothers of boy infants reported their child spent more time outside than girl infants, yet this difference was only significant at 18-months of age. Since 18-months is the age at which the most notable differences in physical activity between boys and girls were shown, it is likely that by providing boy infants with 
more opportunities to be outside where children are known to be more active [41] - either due to cultural or social norms, or perceived ability- mothers are indirectly influencing sex differences in physical activity. Berglind et al., recently found that, in a group of four year old toddlers in Sweden, diurnal patterns of activity were different between boys and girls - with girls being less active and more sedentary than boys particularly when at home with parents, yet this difference was not observed during preschool hours [42]. This finding could lend support to the theory that mothers provide boys with more opportunities to be active than girls. Perhaps this relationship is bidirectional or part of a positive feedback loop, particularly for older infants who have more autonomy around their behaviour decisions - where boy infants may appear to enjoy activities outside more than girls (eg: crying less when outdoors), thus providing positive feedback cues to the mother who in turn reacts by providing more time outside. By being provided with more opportunities to be active, boys may then be presenting with higher activity levels at later stages due to tracking and/or improved motor development.

We also showed differences in physical activity levels and patterns between different developmental stages, as well as age groups. These findings are not surprising, although it is important to note that age differences were found regardless of developmental stage and vice versa. Specifically, time spent in higher intensity physical activities was higher with age. Independent of this relationship, while both walkers and crawlers had higher intensity activity than not yet mobile infants; walkers had the highest intensity activity and this was mostly consistent throughout the day. There was an age effect introduced during the construction of the developmental stages, since questions about walking and crawling were not asked at 3months (and these infants were assumed to be not yet mobile), while questions about walking were not asked at 6-months, and these infants were assumed to be not yet mobile if mothers stated they were not yet crawling. Furthermore, at 18 and 24-months, all infants were assumed to be walking. These assumptions, although valid, limit the conclusions that can be drawn; and the small number of infants reported to be crawling at 6-months and walking at 12-, and 18-months (less than one third in all cases) is concerning in comparison to international norms [32]. However, since the associations between developmental stage and physical activity were significant even after adjusting for age, they are worth considering in future studies. Similar to the current findings, walkers and crawlers were found to have higher activity (even after controlling for carrying) than not yet mobile infants in the Yameogo et al., study; yet the higher activity we observed in crawlers compared to walkers at certain times of day (7 am, $12 \mathrm{pm}$, and $7 \mathrm{pm}$ ) was not observed in the
Yameogo et al., study [40]. The placement of the accelerometer on the wrist in the present study may have resulted in more movement being detected during crawling when the wrist is involved, than during walking. However, since walkers were still more active than crawlers at most times, there is potential that this finding could have also been affected by crawlers being more likely to be carried than walkers, thus limiting their opportunity for movement. Age at commencement of walking has been found to be associated with higher physical activity levels in toddlers in Australia, regardless of how long the toddler had been walking when examined, suggesting an innate effect of early motor development on physical activity levels [19]. Furthermore, earlier infant motor development has been associated with greater sports participation at age 14, suggesting a tracking effect [21]. The current findings are thus important, since it appears that, besides physical activity intensity increasing with age, early attainment of developmental milestones is important for both contemporary and future physical activity. Furthermore, providing opportunities to be active in early infancy (such as tummy time), has been associated with earlier attainment of developmental milestones [43] suggesting a bidirectional effect.

Recent evidence has suggested that physical activity levels in young children differ over the course of the day $[39,42,44]$. The present study has confirmed that infant levels of physical activity follow a similar pattern of accumulation to those observed in older children over the course of the day. Diurnal patterns were able to indicate when infants were accumulating the most activity during the day. The peak in activity seemed to occur between 4 and $6 \mathrm{pm}$ - tending more towards $4 \mathrm{pm}$ in the younger infants and more towards $6 \mathrm{pm}$ in older infants (possibly due to more engagement with family members or opportunities for active play). Smaller peaks occurred around 9 am, with slumps occurring between 12 and $1 \mathrm{pm}$ (likely due to feeding times and/or napping in the younger infants). These patterns are very similar to those observed in 19-month toddlers in Australia, where peaks were seen between 8 and $10 \mathrm{am}$, and between 4 and $5 \mathrm{pm}$, with slumps observed at midday [39]. These diurnal patterns are helpful for the understanding of natural, habitual activity behaviours in infants, which would allow for optimisation of intervention design. Furthermore, understanding normal infant/toddler behaviours allows for comparisons with other infant/toddler populations and may provide a means to determine alterations in normal behaviour signifying illness or other issues. For example, a study which similarly looked at activity patterns in 6-23 month infants/toddlers with severe acute malnutrition, found similar waking times and peaks around 9 am, yet found peak activity to occur quite a bit later than in the current study [40]. This 
could be a cultural or sample specific effect and relationships with health are not clear, yet is worth noting since comparative data in this field is so limited. The authors also found that younger infants were more active than older toddlers during awake hours - which is the converse of the findings from the current study, and indicates shifts in normal behaviours, likely in order to conserve energy for brain and body growth during a period of severe malnutrition [45]. Indeed, Ketcheson et al. recently concluded that examination of daily patterns in physical activity according to age and development in infants are warranted in order to understand differences that may occur in special populations (such as infants with Down's Syndrome) [46].

Since there are no defined physical activity cut-offs for different intensities of activity in infants (although cutoffs for two year olds have been developed [47] these could not be extrapolated to such young infants who are not yet mobile), we examined the entire distribution of physical activity across a broad range of intensities up to $400 \mathrm{mg}$ (where very little time was spent in this sample). This allowed identification of the movement intensities where age and sex differences appear to be greatest, around the $60-70 \mathrm{mg}$ intensity threshold. Using transparent data processing made possible with Axivity AX3 monitors, and describing distribution intensities rather than categorising data according to cut-offs, provides a preliminary movement growth curve against which individual children can be compared. However, further work involving either direct observation or a gold standard measure of energy expenditure would be required to understand what may define high or low intensity activities in infants under age two.

There were very few associations between objectively measured physical activity and maternal reported factors and maternal beliefs. This is the first study to report on maternal beliefs about physical activity and maternal reported activities in infants under two in South Africa. Unlike a study done in 19-month toddlers from Australia [19] that used the same questionnaires as this study, neither time spent with other children, or time spent being active with mother were associated with objectively measured physical activity. In fact, in the present study time spent playing games with an adult was associated with lower physical activity at 6-months of age. This is possibly due to the types of games being played in this age group and setting, which may include sedentary activities such as reading (which are still cognitively beneficial but do not produce high intensity movements). Further qualitative work is thus warranted to contextualise types of activities common in this setting.

Overall time spent restrained was high (average of $161 \mathrm{~min} /$ day for the whole sample), but showed an inverse relationship with age. These findings are logical - as infants become independently mobile, parents are likely to keep them restrained less often. Conversely, overall time spent free to play was low (average $138 \mathrm{~min} /$ day for the whole sample), but showed a direct relationship with age (a similar trend as observed with objectively measured physical activity). These findings are similar to those from an Australian sample of infants, where more unrestricted time was reported with age $(60 \mathrm{~min} /$ day in both Australian infants at 4-month and in South African infants at 3-months, and $180 \mathrm{~min} /$ day in Australian 9month infants vs $150 \mathrm{~min} /$ day in South African 12-month infants) [48]. However unrestricted time was much higher in the Australian toddlers at 20 -months $(420 \mathrm{~min} /$ day $)$ compared to the South African toddlers at 18-months (158 $\mathrm{min} /$ day). Overall restricted time was very similar in the Australian study to those reported in the present study (133 min/day at 4-months in Australia vs $119 \mathrm{~min} /$ day at 3-months in South Africa; $150 \mathrm{~min} /$ day at 9months in Australia vs $154 \mathrm{~min} /$ day at 12 -months in South Africa; and $158 \mathrm{~min} /$ day at 20-months in Australia vs $150 \mathrm{~min} /$ day at 18 -months in South Africa). Interestingly, Australian mothers reported that their infants/toddlers spent much more time being active with a parent than did the South Africa mothers $(43 \mathrm{~min} /$ day at 4months vs $8 \mathrm{~min} /$ day at 3-months; $60 \mathrm{~min} /$ day at 9 -months vs $15 \mathrm{~min} /$ day at 12 -months; and $60 \mathrm{~min} /$ day at 20 -months vs $18 \mathrm{~min} /$ day at 18 -months in Australia vs South Africa respectively) [48]. The patterns shown in the current study suggest that the infants who were offered the most opportunity to be active, were also those who spent the most time in higher intensity activities; but also that South African mothers may not be engaging in enough active play with their infants or providing enough opportunity to be active in comparison to international data.

Only one maternal belief was associated with physical activity: lower concerns about floor play were associated with higher physical activity at 12-months for girls only, suggesting that mothers who were less concerned about their infant being left free to move around on the floor may have provided more opportunity to do so, resulting in higher levels of activity. The fact that this association was only observed for girl infants again points to differential sex specific maternal behaviours and beliefs resulting in providing increased opportunities for physical activity for boy infants, in conjunction with the existence of enhanced safety concerns for girl infants; which could either be due to inherent beliefs or feedback from infant behaviours. Most maternal beliefs about activity were not associated with objectively measured physical activity. It is possible that the questions used in this measurement tool were not well understood in this sample, even though they were piloted and adapted for the Soweto context before use, and internal consistency was acceptable (mean Cronbach alpha $=0.60$ ). It is also possible 
that maternal beliefs about physical activity are not necessarily translating into practice due to constraints of the environment, time availability and family needs, or other barriers that may be preventing mothers from providing sufficient opportunity for play in the Soweto area. Indeed, previous studies in Soweto have shown that young adults report high levels of alcohol and drug abuse, crime, illegal dumping of waste, sewerage issues, road rage, and gang presence [49]- all of which could influence perceived safety for activities such as outdoor play. Furthermore, in a survey of young mothers in Soweto, most mothers lived in households with more than five occupants, and cared for children other than their own biological children, and the majority did not live with the father of their child [22]. These potential barriers definitely require further investigation, in order to better understand the context of these beliefs, and consequent behaviours in such a unique environment.

Most (90\%) infants were meeting the recommended $180 \mathrm{~min}$ of active time as reported by mothers. However, $94 \%$ of infants were not meeting international TV time recommendations of zero minutes of TV time in children under age two [33,34], and mothers reported on average 50 mins in front of the TV per day. TV time was lower in older infants, which, when taken in conjunction with the increased time spent outdoors and higher levels of physical activity in older infants, may be an indication that older infants were substituting TV time with outdoor time and active play. It must be noted that using time in front of a TV as a proxy for TV time has limitations, including the inability to dissociate activities which may be occurring simultaneously (such as sitting in front of the TV whilst playing on the floor which may be better categorised as active time). Nonetheless, exceeding TV time recommendations using this proxy variable was associated with lower motor milestone attainment, and crawlers were the most compliant with guidelines (18\% meeting guidelines) compared to walkers ( $2 \%$ meeting guidelines) and not yet mobile infants ( $4 \%$ meeting guidelines). When treated as a continuous variable, minutes spent watching/in front of the TV was directly associated with BMI z-score in the whole sample, with each $10 \mathrm{~min}$ spent in front of the TV being associated with approximately +0.05 in BMI zscore. This is similar to recent findings from the UK, where $75 \%$ of infants exceeded recommendations [50], and TV time was associated with increased BMI.

This study has several limitations. Firstly, the cross sectional nature of the data limits our ability to determine causality. Furthermore, the assumptions that were made in order to categorise the developmental stages require that the data be interpreted with caution. Some data were not collected at every age (i.e.: maternal beliefs data not collected at 3-, 18-, and 24-months; development and maternal reported physical activity not collected at 24months), resulting in missing data for these questions. Furthermore, the sample size was small when stratifying by age; and findings cannot necessarily be extrapolated to infants outside of South Africa. Lastly, the inability to differentiate between caregiver carrying movements and infant initiated movement limits the interpretation of some of the findings around carrying and objectively measured physical activity, and future work should examine methodological means to deal with this confounding effect. However, the strengths of this study lie in the use of objective measurement of raw acceleration data, which allows full control and transparency of data processing. By examining the distributions of activity across all intensities, we were able to interpret associations that may have been lost if the data were categorised into broader intensity categories.

\section{Conclusions}

In conclusion, this study is the first to show sex differences in the patterns and intensity of physical activity in the first two years of life. Total physical activity was higher in boys, older age groups, and in those with more advanced developmental stages. Children who were provided more opportunities to be active, either through floor play or playing outside, achieved higher levels of activity. Understanding these patterns of activity and the factors associated with them is the first step in being able to determine when and how to intervene effectively. While maternal reported time spent free to be active was sufficient as reported by mothers, and meets minimum recommendations; most infants were spending excessive time in front of a TV, and this TV time was inversely associated with BMI z-score. Thus, providing opportunities to be active through play, and limiting screen time to zero should be encouraged.

\section{Abbreviations}

BMI: Body mass index; CDC: Centre for Disease Control; TV: Television; WHO: World Health Organization

\section{Acknowledgements}

We would like to acknowledge Antonia Smith and Thomas White (MRC Epidemiology Unit, UK) for assistance with the processing of the data, and Lutricia Moagi (MRCMits Developmental Pathways for Health Research Unit, SA) for her assistance with data collection.

Ethics approval and consent to participant:

Ethical approval was obtained from the Human Research Ethics Committee of the University of the Witwatersrand (ethics number M150632). All participants gave written and informed consent for participation in this study.

\section{Funding}

The support of the DST-NRF Centre of Excellence in Human Development at the University of the Witwatersrand, Johannesburg in the Republic of South Africa is hereby acknowledged by AP. Opinions expressed and conclusions arrived at, are those of the author and are not to be attributed to the CoE in Human Development. The work of AP was also supported by the Claude Leon Foundation. The work of SB and KW was supported by UK Medical Research Council (MC_UU_12015/3) and the NIHR Biomedical Research 
Centre Cambridge (IS-BRC-1215-20,014). KDH is supported by an Australian Research Council Future Fellowship (FT130100637), and by the Honorary National Heart Foundation of Australia Future Leader Fellowship (100370). LKM would like to acknowledge funding from the Academy of Medical Sciences-Newton Advanced Fellowship. None of the funders were involved in the design of the study, collection or analysis or interpretation of the data, or in writing of the manuscript.

\section{Availability of data and materials}

The dataset supporting the conclusions of this article is included within the article (and its additional files).

\section{Authors' contributions}

AP was involved in conceptualisation of the manuscript, data collection, cleaning and analysis, and writing of the manuscript. $\mathrm{KDH}$ and $\mathrm{JH}$ were involved in the construction of the questionnaires, conceptualisation of the manuscript, and edited the manuscript. SB and KW were involved in conceptualisation of the manuscript, processing of the data and edited the manuscript. LKM conceptualised the project and edited the manuscript. All authors read and approved the final manuscript.

\section{Consent for publication}

Not applicable.

\section{Competing interests}

The authors declare that they have no competing interests.

\section{Publisher's Note}

Springer Nature remains neutral with regard to jurisdictional claims in published maps and institutional affiliations.

\author{
Author details \\ ${ }^{1}$ MRCMITS Developmental Pathways for Health Research Unit, Department \\ of Paediatrics, School of Clinical Medicine, Faculty of Health Sciences, \\ University of Witwatersrand, Johannesburg, South Africa. ${ }^{2}$ MRC Epidemiology \\ Unit, University of Cambridge, Cambridge, UK. ${ }^{3}$ Institute for Physical Activity \\ and Nutrition, Deakin University, Geelong, Australia.
}

\section{Received: 28 August 2017 Accepted: 11 December 2017} Published online: 22 December 2017

\section{References}

1. van der Velde JH, Savelberg HH, Schaper NC, Koster A. Moderate activity and fitness, not sedentary time, are independently associated with cardiometabolic risk in U.S. adults aged 18-49. Int J Environ Res Public Health. 2015;12(3):2330-43.

2. Timmons BW, Leblanc AG, Carson V, Connor Gorber S, Dillman C, Janssen I, et al. Systematic review of physical activity and health in the early years (aged 0-4 years). Appl Physiol Nutr Metab. 2012;37(4):773-92.

3. LeBlanc AG, Spence JC, Carson V, Connor Gorber S, Dillman C, Janssen I, et al. Systematic review of sedentary behaviour and health indicators in the early years (aged 0-4 years). Appl Physiol Nutr Metab. 2012;37(4):753-72.

4. Downing KL, Hnatiuk J, Hesketh KD. Prevalence of sedentary behavior in children under 2years: a systematic review. Prev Med. 2015;78:105-14.

5. Araujo de Franca GV, Restrepo-Mendez MC, Loret de Mola C, Victora CG. Size at birth and abdominal adiposity in adults: a systematic review and meta-analysis. Obes Rev. 2014;15(2):77-91.

6. Prioreschi A, Micklesfield LK. A scoping review examining physical activity measurement and levels in the first 2 years of life. Child Care Health Dev. 2016;42(6):775-83.

7. Prioreschi A, Wrottesley SV, Draper CE, Tomaz SA, Cook CJ, Watson ED, et al. Maternal and early life nutrition and physical activity: setting the research and intervention agenda for addressing the double burden of malnutrition in South African children. Global Health Action. 2017;10(1):1301085.

8. Shisana O, Labadarios D, Rehle T, Simbayi L, Zuma K, Dhansay A, et al. South African National Health and nutrition examination survey (SANHANES-1): 2014 edition. Cape Town: HSRC Press; 2014

9. Uys M, Bassett S, Draper CE, Micklesfield L, Monyeki A, de Villiers A, et al. Results from South Africa's 2016 report card on physical activity for children and youth. J Phys Act Health. 2016;13(11 Suppl 2):S265-73.
10. Campbell KJ, Lioret S, McNaughton SA, Crawford DA, Salmon J, Ball K, et al. A parent-focused intervention to reduce infant obesity risk behaviors: a randomized trial. Pediatrics. 2013;131(4):652-60.

11. Taylor BJ, Heath AL, Galland BC, Gray AR, Lawrence JA, Sayers RM, et al. Prevention of overweight in infancy (POI.Nz) study: a randomised controlled trial of sleep, food and activity interventions for preventing overweight from birth. BMC Public Health. 2011;11:942.

12. Jones S, Hendricks S, Draper CE. Assessment of physical activity and sedentary behavior at preschools in cape town, South Africa. Child Obes. 2014;10(6):501-10.

13. Hinkley T, Crawford D, Salmon J, Okely AD, Hesketh K. Preschool children and physical activity: a review of correlates. Am J Prev Med. 2008;34(5):435-41.

14. Hesketh KR, O'Malley C, Paes VM, Moore H, Summerbell C, Ong KK, et al. Determinants of change in physical activity in children 0-6 years of age: a systematic review of quantitative literature. Sports Med. 2017:47(7):1349-74.

15. Faurholt-Jepsen D, Hansen KB, van Hees VT, Christensen LB, Girma T, Friis H, et al. Children treated for severe acute malnutrition experience a rapid increase in physical activity a few days after admission. J Pediatr. 2014;164(6):1421-4.

16. Pulakka A, Cheung YB, Maleta K, Dewey KG, Kumwenda C, Bendabenda J, et al. Effect of 12-month intervention with lipid-based nutrient supplement on the physical activity of Malawian toddlers: a randomised, controlled trial. $\mathrm{Br}$ J Nutr. 2017;117(4):511-8.

17. Duch $\mathrm{H}$, Fisher EM, Ensari I, Harrington A. Screen time use in children under 3 years old: a systematic review of correlates. Int J Behav Nutr Phys Act. 2013;10:102. doi:10.1080/16549716.2017.1301085.

18. Certain LK, Kahn RS. Prevalence, correlates and trajectory of television viewing among infants and toddlers. Paediatrics. 2002;109(4):634-42.

19. Hnatiuk J, Salmon J, Campbell KJ, Ridgers ND, Hesketh KD. Early childhood predictors of toddlers' physical activity: longitudinal findings from the Melbourne InFANT program. Int J Behav Nutr Phys Act. 2013;10:123.

20. Hager ER, Gormley CE, Latta LW, Treuth MS, Caulfield LE, Black MM. Toddler physical activity study: laboratory and community studies to evaluate accelerometer validity and correlates. BMC Public Health. 2016;16:936.

21. Ridgway $C L$, Ong KK, Tammelin TH, Sharp S, Ekelund U, Jarvelin MR. Infant motor development predicts sports participation at age 14 years: northern Finland birth cohort of 1966. PLoS One. 2009;4(8):e6837.

22. Jordan N, Patel L, Hochfeld T. Early motherhood in Soweto: the nexus between the child support grant and developmental social work services. Soc Work. 2014:50(3):392-409.

23. White T, Westgate K, Wareham NJ, Brage S. Estimation of physical activity energy expenditure during free-living from wrist Accelerometry in UK adults. PLoS One. 2016;11(12):e0167472.

24. Doherty A, Jackson D, Hammerla N, Plotz T, Olivier P, Granat MH, et al. Large scale population assessment of physical activity using wrist worn accelerometers: the UK biobank study. PLoS One. 2017;12(2):e0169649.

25. Prioreschi A, Brage S, Nappey T, Westgate K, Olivier P, Micklesfield LK. Design and feasibility of an infant wearable band for the measurement of physical activity. Under review at BMC Pilot and Feasibility Studies; 2017.

26. van Hees VT, Fang Z, Langford J, Assah F, Mohammad A, da Silva IC, et al. Autocalibration of accelerometer data for free-living physical activity assessment using local gravity and temperature: an evaluation on four continents. J Appl Physiol (1985). 2014;117(7):738-44.

27. van Hees VT, Gorzelniak L, Dean Leon EC, Eder M, Pias M, Taherian S, et al Separating movement and gravity components in an acceleration signal and implications for the assessment of human daily physical activity. PLoS One. 2013;8(4):e61691.

28. Brage S, Westgate K, Wijndaele K, Godinho J, Griffin S, Wareham N. Evaluation of a method for minimising diurnal information bias in objective sensor data. Amherst: ICAMPAM Conference Proceeding; 2013.

29. Pitchford EA, Ketcheson LR, Kwon HJ, Ulrich DA. Minimum accelerometer wear time in infants: a generalizability study. J Phys Act Health. 2017;14:421-8.

30. de Onis MWHO. Child growth standards based on length/height, weight and age. Acta Paediatr. 2006;95:76-85.

31. WHO Anthro for personal computers, version 3.2.2, 2011: Software for assessing growth and development of the world's children. Geneva:WHO, 2010: (http://www.who.int/childgrowth/software/en/ ).

32. WHO Multicentre growth reference study group. WHO motor development study: windows of achievement for six gross motor development milestones. Acta Paediatr. 2006:450:86-95.

33. Tremblay MS, Leblanc AG, Carson V, Choquette L, Connor Gorber S, Dillman $\mathrm{C}$, et al. Canadian physical activity guidelines for the early years (aged 0-4 years). Applied Physiology Nutrition and Medicine. 2012;37(2):345-56. 
34. Australian Government Department of Health \& Ageing. Get up \& grow: healthy eating and physical activity for early childhood. Canberra: Commonwealth of Australia; 2009.

35. da Silva IC, van Hees VT, Ramires W, Knuth AG, Bielemann RM, Ekelund U, et al. Physical activity levels in three Brazilian birth cohorts as assessed with raw triaxial wrist accelerometry. Int J Epidemiol. 2014;43(6):1959-68.

36. Van Cauwenberghe E, Gubbels J, De Bourdeaudhuij I, Cardon G. Feasibility and validity of accelerometer measurements to assess physical activity in toddlers. Int J Behav Nutr Phys Act. 2011;8:67.

37. Johansson E, Mei H, Xiu L, Svensson V, Xiong Y, Marcus C, et al. Physical activity in young children and their parents-an early STOPP Sweden-China comparison study. Sci Rep. 2016;6:29595.

38. Wijtzes Al, Kooijman MN, Kiefte-de Jong JC, de Vries Sl, Henrichs J, Jansen W, et al. Correlates of physical activity in 2-year-old toddlers: the generation R study. J Pediatr. 2013;163(3):791-9. e791-792

39. Hnatiuk J, Ridgers ND, Salmon J, Campbell K, McCallum Z, Hesketh K. Physical activity levels and patterns of 19-month-old children. Med Sci Sports Exerc. 2012;44(9):1715-20.

40. Yameogo CW, Cichon B, Fabiansen C, luel-Brockdorf AS, Shepherd S, Filteau $\mathrm{S}$, et al. Correlates of physical activity among young children with moderate acute malnutrition. J Pediatr. 2017;181:235-41.

41. Larouche R, Garriguet D, Tremblay MS. Outdoor time, physical activity and sedentary time among young children: the 2012-2013 Canadian health measures survey. Can J Public Health. 2017:107(6):500-6.

42. Berglind D, Tynelius P. Objectively measured physical activity patterns, sedentary time and parent-reported screen-time across the day in four-yearold Swedish children. BMC Public Health. 2017;18(1):69.

43. Gross RS, Mendelsohn AL, Yin HS, Tomopoulos S, Gross MB, Scheinmann R, et al. Randomized controlled trial of an early child obesity prevention intervention: impacts on infant tummy time. Obesity (Silver Spring). 2017; 25(5):920-7.

44. Hesketh K, McMinn A, Ekelund U, Sharp S, Collings P, Harvey N, et al. Objectively measured physical activity in four-year-old British children: a cross sectional analysis of activity patterns segmented across the day. Int J Behav Nutr Phys Act. 2014;11(1). doi:10.1186/1479-5868-11-1.

45. Rising R, Sonmez GT. Energy expenditure and physical activity in recovering malnourished infants. J Nutr Metab. 2010; doi:10.1155/2010/171490.

46. Ketcheson L, Pitchford EA, Kwon HJ, Ulrich DA. Physical activity patterns in infants with and without down syndrome. Pediatr Phys Ther. 2017:29(3):200-6.

47. Johansson E, Ekelund U, Nero H, Marcus C, Hagströmer M. Calibration and cross-validation of a wrist-worn Actigraph in young preschoolers. Pediatric Obesity. 2014;10:1-6.

48. Hesketh KD, Crawford DA, Abbott G, Campbell KJ, Salmon J. Prevalence and stability of active play, restricted movement and television viewing in infants. Early Child Dev Care. 2014;185(6):883-94.

49. Pradeilles R, Rousham E, Norris S, Griffiths PL. Urban south African adolescents' perceptions of their neighborhood socio economic environments: the birth to twenty plus cohort study. Children, Youth and Environments. 2014;24(3):173-200

50. Barber SE, Kelly B, Collings PJ, Nagy L, Bywater T, Wright J. Prevalence, trajectories, and determinants of television viewing time in an ethnically diverse sample of young children from the UK. Int J Behav Nutr Phys Act. 2017;14(1):88.

\section{Submit your next manuscript to BioMed Central and we will help you at every step:}

- We accept pre-submission inquiries

- Our selector tool helps you to find the most relevant journal

- We provide round the clock customer support

- Convenient online submission

- Thorough peer review

- Inclusion in PubMed and all major indexing services

- Maximum visibility for your research

Submit your manuscript at www.biomedcentral.com/submit

) Biomed Central 